Recepción: 17 / 08 / 2018

Aceptación: 15 / 09 / 2018

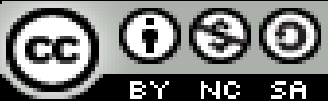

Ciencias de la computación

Publicación: 01 / 11 / 2018

Artículo de investigación

\title{
Matlab como herramienta de análisis estadístico
}

\section{Matlab as a statistical analysis tool}

\section{Matlab como ferramenta de análise estatística}

\author{
Alexandra O. Pazmiño-Armijos ${ }^{\mathrm{I}}$ \\ apazmino_a@espoch.edu.ec \\ Jairo R. Jácome-Tinoco II \\ jjacome@espoch.edu.ec \\ Paulina F. Bolaños-Logroño ${ }^{\text {III }}$ \\ paulina.bolanos@espoch.edu.ec \\ Cristian G. Santiana-Espín ${ }^{\text {IV }}$ \\ santiana@espoch.edu.ec
}

Correspondencia: apazmino_a@espoch.edu.ec

\footnotetext{
I Magíster en Informática Empresarial, Especialista en Redes de Comunicación de Datos, Ingeniera en Electrónica y Computación, tecnólogo en Informática Aplicada, Docente de la Escuela Politécnica de Chimborazo ESPOCH, Riobamba, Ecuador.

${ }^{\text {II }}$ Magíster en Sistemas de Telecomunicaciones, Ingeniero en Electrónica y Computación, Tecnólogo en Informática Aplicada, Docente de la Escuela Politécnica de Chimborazo ESPOCH, Riobamba, Ecuador.

III Magíster en Sistemas de Control y Automatización Industrial, Ingeniera en Electrónica y Computación, Tecnóloga en Informática Aplicada, Docente de la Escuela Politécnica de Chimborazo ESPOCH, Riobamba, Ecuador.

${ }^{\text {IV }}$ Magíster en Formulación, Evaluación y Gerencia de Proyectos para el Desarrollo, Magíster en Sistemas de Control y Automatización Industrial, Ingeniero en Electrónica Control y Redes Industriales, Docente de la Escuela Politécnica de Chimborazo ESPOCH, Riobamba, Ecuador.
} 


\title{
Resumen
}

El presente artículo estudia la caracterización del software MATLAB como herramienta de apoyo para el análisis estadístico de datos de una investigación. Para ello, se aplicó una metodología de tipo descriptivo analítica y de campo bajo un enfoque cuantitativo, con una estadística descriptiva inferencial al conjunto de datos sometidos al análisis. La población objeto de estudio estuvo constituido por 52 estudiantes de la carrera de ingeniería en sistemas y en proceso de elaboración de sus tesis de grado, con una muestra de tipo aleatoria. Para la obtención de los datos e información se utilizó la técnica de la encuesta a través de un instrumento tipo cuestionario y una entrevista semi - estructurada. Los resultados indicaron que el 90\% de los investigados tienen conocimiento del software integrador "MATLAB", el 72\% poseen interés en manejarlo y solo el $51 \%$ de los docentes lo usan. Entre sus conclusiones se pudo constatar un alto interés por el uso y manejo del software por parte de los investigados para resolver la estadística de los datos e información de sus investigaciones.

Palabras clave: MATLAB; análisis estadístico; datos; investigación.

\begin{abstract}
This article studies the characterization of MATLAB software as a support tool for the statistical analysis of research data. For this, an analytical and field descriptive methodology was applied under a quantitative approach, with a descriptive statistic inferential to the set of data submitted to the analysis. The study population consisted of 52 students from the engineering career in systems and in the process of preparing their theses, with a sample of random type. To obtain the data and information, the survey technique was used through a questionnaire - type instrument and a semi - structured interview. The results indicated that $90 \%$ of those researched have knowledge of the integrating software "MATLAB", 72\% have an interest in managing it and only $51 \%$ of teachers use it. Among its conclusions, it was possible to observe a high interest in the use and management of the software by the researchers to solve the statistics of the data and information of their investigations.
\end{abstract}

Keywords: MATLAB; statistic analysis; data; investigation. 


\section{Resumo}

Este artigo estuda a caracterização do software MATLAB como ferramenta de suporte para a análise estatística de dados de pesquisa. Para isso, aplicou-se uma metodologia analítica e descritiva de campo sob abordagem quantitativa, com uma estatística descritiva inferencial ao conjunto de dados submetidos à análise. A população do estudo foi composta por 52 estudantes da carreira de engenharia em sistemas e no processo de elaboração de suas teses, com uma amostra do tipo aleatória. Para obtenção dos dados e informações, utilizou - se a técnica de pesquisa por meio de instrumento tipo questionário e entrevista semi - estruturada. Os resultados indicaram que $90 \%$ dos pesquisados possuem conhecimento do software integrador "MATLAB", $72 \%$ têm interesse em gerenciá-lo e apenas $51 \%$ dos professores o utilizam. Entre suas conclusões, foi possível observar um alto interesse no uso e gerenciamento do software pelos pesquisadores para resolver as estatísticas dos dados e informações de suas investigações.

Palavras chave: MATLAB; análise estatística; dados; investigação.

\section{Introducción}

MATLAB fue creado por Cleve Moler en 1984, surgiendo la primera versión con la idea de emplear paquetes de subrutinas escritas en Fortran en los cursos de álgebra lineal y análisis numérico, sin necesidad de escribir programas en dicho lenguaje. Aunque Moler comenzó MATLAB, con estrechos problemas relacionados con las matemáticas en la mente, el lenguaje en última instancia resultó influyente en una amplia gama de campos. En particular, Moler dijo "Computerworld", poco se volvió MATLAB en un lenguaje de programación amplia, con amplias implicaciones. En poco tiempo, el software MATLAB comenzó a ser utilizado en una gran variedad de áreas, y el área académica no fue la excepción.

Entendiéndolo como una herramienta de software matemático que ofrece un entorno de desarrollo integrado (IDE) con un lenguaje de programación propio (lenguaje M). Está disponible para las plataformas Unix, Windows, Mac OS X ylGNU/Linux. Entre sus prestaciones básicas se hallan: la manipulación de matrices, la representación de datos y funciones, la implementación de algoritmos, la creación de interfaces de usuario (GUI) y la comunicación con programas en otros lenguajes y con otros dispositivos hardware (http://es.wikipedia.org/wiki/MATLAB, 2014) 
Es de indicar, que MATLAB es ampliamente conocido y utilizado en universidades e institutos para el aprendizaje de cursos básicos y avanzados de matemáticas, ciencias y especialmente, ingeniería. MATLAB es un programa de cálculo numérico orientado a matrices, por lo tanto, de procesamiento y análisis estadístico de datos en pro de gran utilidad. Es de destacar que el análisis del uso de esta herramienta en la estadística, facilita la representación de los datos en términos de vectores, matrices y archivos, facilitando la visualización y comprensión del comportamiento de un problema estadístico.

Si bien los estudios sobre este tema en el ámbito educativo son considerables, no existen aquellos que abordan cómo el MATLAB es un apoyo para la comprensión en los temas relacionados con vectores y matrices. MATLAB es ampliamente conocido y utilizado en universidades e institutos para el aprendizaje de cursos básicos y avanzados de matemáticas, ciencias y especialmente, ingeniería. (Wiley, 2005).

Por lo tanto, el objetivo de este trabajo entonces es, indagar como el software integrador MATLAB como herramienta de apoyo en la materia de enseñanza de Algoritmos y lenguajes de programación ayuda a los tesistas en el análisis y compresión de los datos aportados por su investigación. El logro de este artículo se direcciono a partir de la caracterización del uso del software MATLAB como herramienta de apoyo para el análisis estadístico de datos. Según García (2005), las técnicas adicionales y herramientas de programación, que presenta MATLAB, en la obtención de análisis de los datos de una investigación de manera eficiente y eficaz permiten la comprensión y valoración de la tecnología para la solución de problemas de la vida cotidiana.

\section{Desarrollo}

Matlab es un software muy usado en universidades y centros de investigación y desarrollo. En los últimos años ha aumentado el número de prestaciones, como la de programar directamente procesadores digitales de señal o crear código VHDL.

En 2004, se estimaba que MATLAB era empleado por más de un millón de personas en ámbitos académicos y empresariales. 
Entre sus prestaciones básicas se hallan: la manipulación de matrices, la representación de datos y funciones, la implementación de algoritmos, la creación de interfaces de usuario (GUI) y la comunicación con programas en otros lenguajes y con otros dispositivos hardware.

El paquete MATLAB dispone de dos herramientas adicionales que expanden sus prestaciones, a saber, Simulink (plataforma de simulación multidominio) y GUIDE (editor de interfaces de usuario - GUI). Además, se pueden ampliar las capacidades de MATLAB con las cajas de herramientas (toolboxes); y las de Simulink con los paquetes de bloques (blocksets).

El software de simulación. Ayuda a predecir el comportamiento de un sistema. El software de simulación se puede utilizar para evaluar un diseño nuevo, diagnosticar problemas de un diseño existente y probar un sistema en condiciones que son difíciles de reproducir, como por ejemplo un satélite en el espacio exterior. Para ejecutar una simulación, se necesita un modelo matemático del sistema, que se puede expresar como un diagrama de bloques, un esquema, un diagrama de estados o incluso código. El software de simulación calcula el comportamiento del modelo a medida que las condiciones evolucionan con el tiempo o a medida que se producen eventos. El software de simulación también incluye herramientas de visualización, tales como data displays y animación 3D, para contribuir a supervisar la simulación a medida que se ejecuta.

Los ingenieros y los científicos utilizan el software de simulación por diversas razones:

- Crear y simular modelos es más barato que construir y probar prototipos de hardware.

- Se puede utilizar el software de simulación para probar distintos diseños antes de crear uno en hardware.

- Es posible conectar el software de simulación al hardware para probar la integración del diseño completo.

Es de indicar que MATLAB contribuye a simplificar tareas que, de otra forma, resultarían lentas, tales como:

- Limpieza de datos que presenten errores, valores atípicos o duplicados

- Gestión de datos ausentes mediante descarte, filtrado o imputación

- Eliminación del ruido de los datos de sensores con técnicas avanzadas de procesamiento de señales 
- Fusión y alineación temporal de los datos con diferentes tasas de muestreo

- Selección de características para reducir datos con un número elevado de dimensiones a fin de aumentar el poder de predicción del modelo

- Extracción y transformación de características para reducir la dimensionalidad

- Análisis del dominio, como procesamiento de señales, imagen y vídeo

\section{La estadística de los datos en la investigación científica}

La estadística en una investigación, implica coordinar experiencias previas, conocimiento e intuición para encontrar la solución adecuada. Un investigador para resolver la estadística de los datos de su trabajo, la formula en sus propios términos, experimenta, observa, tantea, conjetura y valida (Parra, 2001). Para ello, hay cuatro momentos fundamentales según George Pólya (2001), tal como se describen:

Entender el problema: se refiere a que el estudiante pueda responderse una serie de preguntas en la que sea capaz de identificar la incógnita, los datos y las condiciones del problema de investigación, entre la información y datos obtenidos de sus investigados.

Configurar el plan: se refiere al cómo o qué estrategia va a usar el estudiante para presentar los resultados de la información obtenida. Las estrategias pueden partir desde aplicar pruebas de ensayo y error, hasta plantear toda una táctica que le permita intentar llegar a la solución del mismo a partir de los distintos softwares que se ofrecen para tal fin.

Ejecutar el plan: se refiere a la puesta en práctica de lo que el estudiante estableció en la configuración. En este punto puede suceder que en un momento determinado lo que se planteó no sea pertinente para el análisis de la información, razón por la cual hay que replantear la estrategia y volver a comenzar. Generalmente en la ejecución se usan procesos matemáticos que permitan darle la exactitud que requiere a los datos procesados.

Examinar la solución: se refiere al poderse cuestionar sobre lo que se hizo, ver si el proceso desarrollado permitió en realidad resolver lo requerido por el objetivo de la investigados y la función de los datos e información obtenida. En este paso el estudiante debe acudir a sus procesos metacognitivos para revisar si lo que hizo está bien o está mal y, si es necesario, replantear el proceso de resolución. 


\section{Los problemas estadísticos de una investigación}

Según Pozo y Gómez (2000), se pueden distinguir cuatro tipos de problemas estadísticos.

- Problemas estadísticos. Tarea concreta para la que se recogen datos que posteriormente son analizados estadísticamente.

- Problemas de Información. Se pretende describir exhaustivamente el comportamiento de una variable.

- Problemas No experimentales. Estudian la relación entre dos variables. El estudiante analiza por ejemplo como se distribuyen los sujetos entre los grupos de análisis. No hay ninguna interpretación causal de las posibles conclusiones, excepto que si por razones teóricas se puede justificar la homogeneidad de los grupos estudiados.

- Problemas experimentales. Estudian la relación entre variables controladas y unas aleatorias. Si la manipulación adecuada de las variables controladas genera diferencia en los valores de la variable aleatoria es factible una conclusión de carácter causal.

\section{Metodología}

El presente artículo siguió una metodología de tipo descriptivo analítica y de campo (Hernández et al., 2014). Se aplicó un enfoque cuantitativo, al utilizar la estadística descriptiva inferencial al conjunto de datos sometidos al análisis, al caracterizar el software MATLAB como herramienta de apoyo para el análisis estadístico de datos. La población objeto de estudio estuvo constituido por 52 estudiantes en proceso de elaboración de sus tesis de grado y la muestra fue de tipo aleatoria. Para la obtención de los datos e información se utilizó la técnica de la encuesta a través de un instrumento tipo cuestionario y una entrevista semi - estructurada.

\section{Resultados}

Para la obtención de resultados en este artículo, se utilizó la técnica de la encuesta a través de un instrumento tipo cuestionario y una entrevista semi - estructurada, aplicados a 52 estudiantes en proceso de elaboración de sus tesis de grado, de la carrera ingeniería de sistemas, los mismos se presentan en tablas con sus respectivos gráficos. 


\section{Tabla 1}

Estadística descriptiva sobre del conocimiento del software integrador "MATLAB"

\begin{tabular}{ccccc}
\hline ANÁLISIS/ ALTERNATIVAS & FR & \% & FRA & \% A \\
\hline Conocen & 47 & 90 & 47 & 23 \\
No conocen & 5 & 10 & 52 & 73 \\
No respondió & 0 & 0 & 0 & 100 \\
TOTAL & 52 & 100 & 52 & 100 \\
\hline \hline
\end{tabular}

Fuente: Elaboración propia (2018).

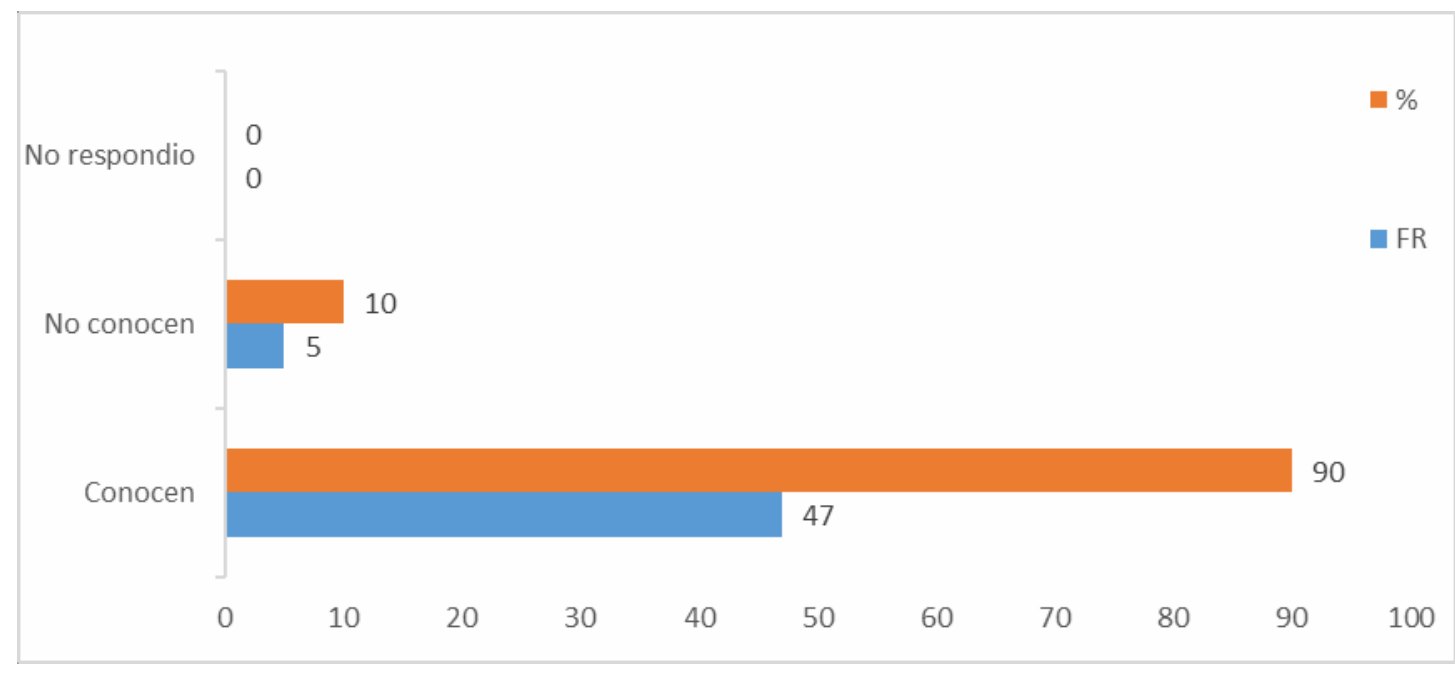

Grafico 1: Estadística descriptiva sobre del conocimiento del software integrador "MATLAB" Fuente: Elaboración propia (2018).

El 90\% indicó que conocían el software integrador "MATLAB", como herramienta matemática y cuya prestación básica estaban dirigidas específicamente para el abordaje de la manipulación de matrices e implementación de algoritmos, desconociendo su utilidad en la representación de datos y funciones. Seguido del $10 \%$, que indicaron la alternativa no lo conocen y para la alternativa no respondió no hubo opinión alguna.

Tabla2 
Estadística descriptiva del interés por manejar el software integrador "MATLAB"

\begin{tabular}{ccccc}
\hline ANÁLISIS/ ALTERNATIVAS & FR & \% & FRA & \% A \\
\hline Alto interés & 37 & 72 & 37 & 72 \\
Mediano interés & 13 & 25 & 50 & 97 \\
Sin interés & 2 & 3 & 52 & 100 \\
TOTAL & 52 & 100 & 52 & 100 \\
\hline \hline
\end{tabular}

Fuente: Elaboración propia (2018).

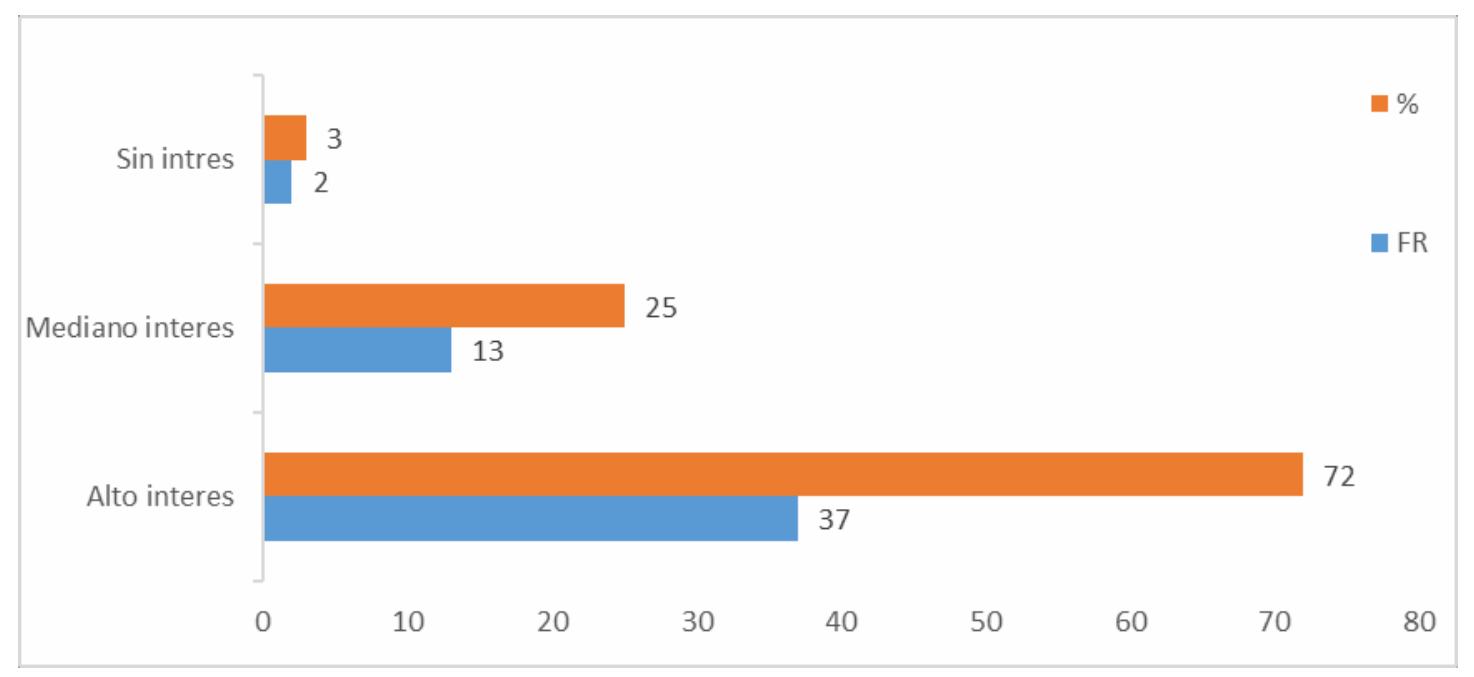

Grafico 2: Estadística descriptiva del interés por manejar el software integrador "MATLAB" Fuente: Elaboración propia (2018).

Existe alto interés de su manejo manifestado por el $72 \%$ de los estudiantes investigados, como herramienta estadística de interpretación de sus datos e informaciones obtenidas posterior al proceso de aplicación de los instrumentos de investigación utilizados. Seguido del 25\% que indicaron la alternativa mediano interés por conocerlo y para la alternativa sin interés el 3\% del total de ellos. 


\section{Tabla3}

Estadística descriptiva del uso docente del software integrador "MATLAB"

\begin{tabular}{ccccc}
\hline ANÁLISIS/ ALTERNATIVAS & FR & \% & FRA & \% A \\
\hline Alto uso & 3 & 4 & 3 & 4 \\
Mediano uso & 23 & 45 & 26 & 49 \\
Sin uso & 26 & 51 & 52 & 100 \\
TOTAL & 52 & 100 & 52 & 100 \\
\hline \hline
\end{tabular}

Fuente: Elaboración propia (2018).

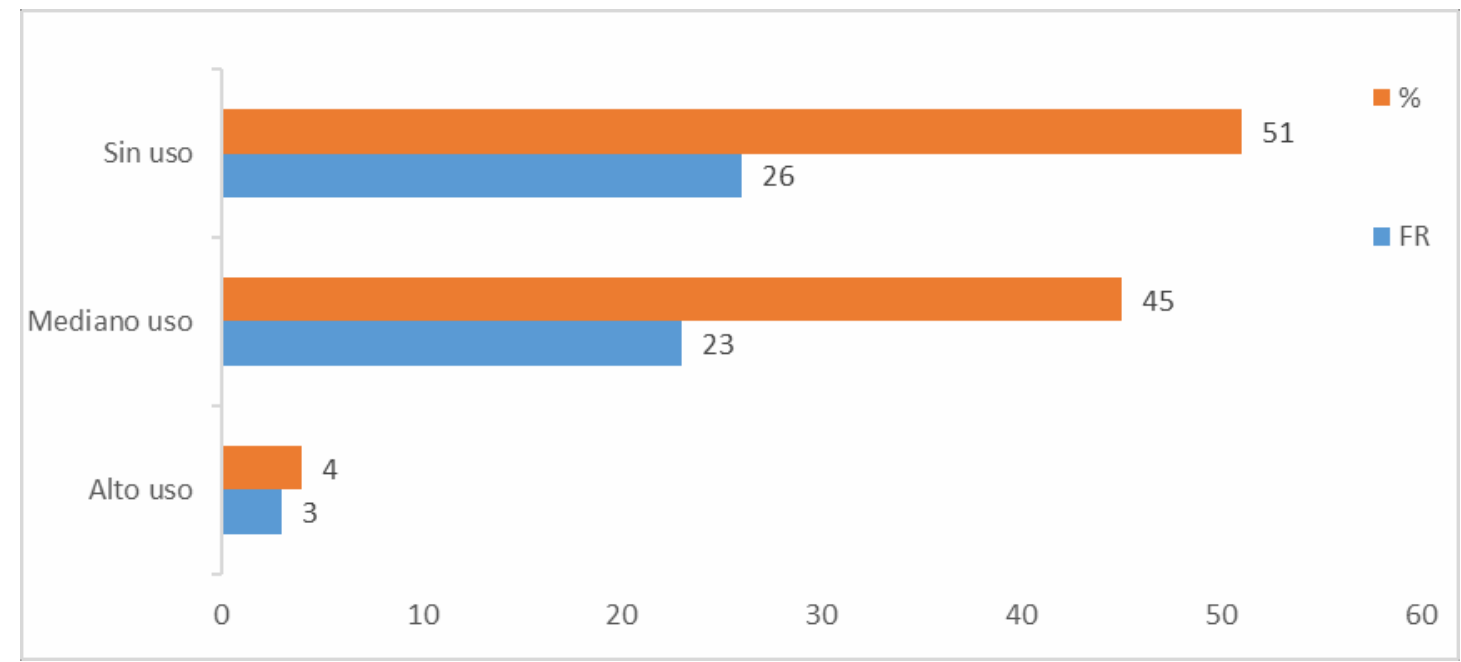

Grafico 3: Estadística descriptiva del uso docente del software integrador "MATLAB"

Fuente: Elaboración propia (2018).

El 51\% de los alumnos tesistas investigados, afirmaron que el software integrador MATLAB no es usado por los docentes en las cátedras que se requiere programar, exaltando el uso del software DevC++. Seguido del 45\%, que indicaron la alternativa mediano uso y para la alternativa alto uso solo el 4\% de las opiniones.

\section{Conclusiones}

Se pudo evidenciar que, existe un alto porcentaje de alumnos conocedores de la existencia del software integrador "MATLAB. Sin embargo, se indicó su desconocimiento en relación a su utilidad como medio de análisis de datos estadísticos para presentar los resultados de los datos e informaciones de una investigación. Por otro lado, se pudo constatar un alto interés por parte de 
los alumnos en proceso de elaboración de tesis para manejarlo. Asimismo, expusieron que existe el uso, con mayor frecuencia, por parte de los docentes del software como el DevC++, a pesar de ser de mayor dificultad de manejo que el MATLAB.

\section{Referencias bibliográficas}

García de Jalón J., R. J. (2005). Matlab como herramienta de apoyo para el rendimiento académico. Recuperado en: www.eumed.net/rev/tectzapic/2015/01/matlab.html

Hernández R., Fernández C. y Baptista P. (2014). Metodología de la investigación. Estado de México: Editorial Mc Graw Hill. p.p. 80, 146.

Parra B. (2001). Dos concepciones de resolución de problemas de Matemáticas. A. Arriaga y H. Barrón (compiladores): La enseñanza de las Matemáticas en la escuela secundaria (p.p. 13-32).

Pólya, G. (2001). Mathematical discovery. On Understanding, Learning and Teaching Problem Solving. Nueva York: John Wiley \& Sons.

Pozo J y Gómez M. (2000). La solución de problemas en Ciencias de la Naturaleza. Madrid. Wiley, J. (2005). MATLAB: An introduction Whit Aplications. Hoboken (NJ), USA: REVERTE S.A. 